\title{
Charge representation of a small two-dimensional Josephson-junction array in the quantum regime
}

\author{
P. Lafarge, M. Matters, and J. E. Mooij \\ Department of Applied Physics and Delft Institute for Microelectronics and Submicron Technology, Delft University of Technology, \\ P.O. Box 5046, 2600 GA Delft, The Netherlands
}

(Received 12 October 1995; revised manuscript received 22 April 1996)

\begin{abstract}
Using the charge representation, we calculate the ground state energy and the critical current of a small two-dimensional Josephson junction array subject to both charge and magnetic frustration. In the quantum regime the ground state of the array is a superposition of charge states, allowing a supercurrent to flow through the circuit. Both the ground state energy and the critical current can be tuned by the two frustrations. We show that the notion of a vortex is compatible with a charge representation of the array. [S0163-1829(96)00933-2]
\end{abstract}

Recent experiments ${ }^{1-4}$ have demonstrated the effects of the competition between tunable Josephson and charging effects in small arrays of superconducting tunnel junctions. The interplay of these phenomena is a direct consequence of the conjugation relation between phase and number-ofparticle variables in a superconductor. The two characteristic energies involved are the Josephson coupling energy $E_{J}$ and the charging energy $E_{c}=e^{2} / 2 C$, where $C$ is the junction capacitance. In the quantum regime, they are comparable and therefore both the charge and phase degrees of freedom of the array are quantum variables. The dynamics of a quantum array can be described either in terms of vortices or in terms of Cooper pairs. Implications of this duality have been investigated in the limit of infinite arrays. ${ }^{5,6}$ However, for small two-dimensional arrays, ${ }^{7}$ the link between the two descriptions is not clearly established and a quantum description is needed.

In this paper we consider the small two-dimensional array depicted in Fig. 1. It consists of two superconducting electrodes, or islands, connected to each other and to the leads by small Josephson junctions with $E_{J} \lesssim E_{c}$. From the phase

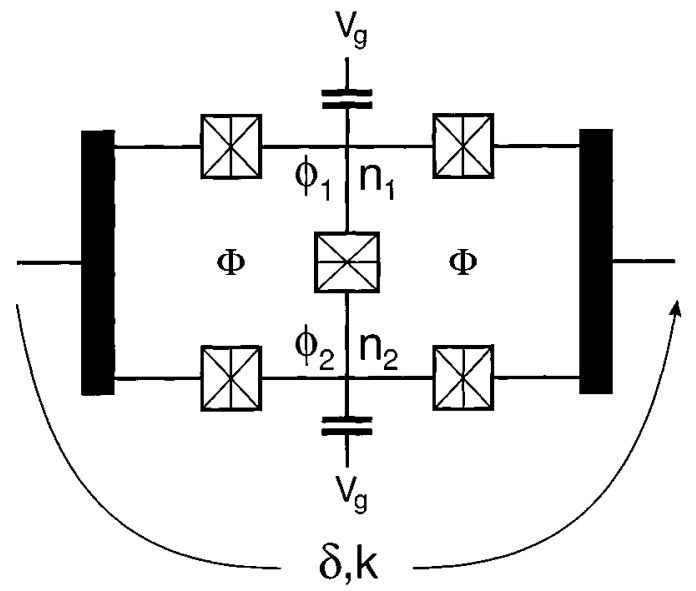

FIG. 1. Schematic of the small Josephson junction array. The two islands are subject to a gate-induced charge $C_{g} V_{g}$ and the two loops to a magnetic flux $\Phi$. The junctions connecting the islands to the leads are labeled from 1 (upper left) to 4 (lower right). The middle junction connecting the two islands is junction 5 . point of view, this circuit can be seen as an array made of two loops. This is indeed the simplest array, exhibiting in a symmetric way the characteristic features of the quantum regime. The Josephson coupling across the junctions, which can be tuned by an applied magnetic flux, tends to fix the phase of the superconducting wave function of the islands and allows a supercurrent to flow through the device. On the other hand, the electrostatic energy reduces the fluctuations of the charge on the islands. By means of a gate voltage one can vary the electrostatic energy required to change the number of Cooper pairs on the islands and therefore the critical current. The presence of only two islands makes it experimentally feasible to apply a uniform gate-induced charge to the array, unlike in a larger array where it is practically impossible to compensate for all the random offset charges caused by impurities in the underlying substrate or in the tunnel barrier. Experiments in small arrays are usually described in terms of vortex motion while the charge representation has been used so far only for treating the circuit made of two small Josephson junctions in series. The latter has been extensively studied both theoretically ${ }^{8-10}$ and experimentally,3,11-14 and its behavior is now well understood. ${ }^{15}$ Here, we extend the charge representation developed for the double junction to the case of a small twodimensional array. We compute the ground state energy and the critical current as a function of induced charge and applied magnetic flux.

All the junctions in the array are identical with a Josephson coupling energy $E_{J}$ and a capacitance $C$. It has been shown that, at zero temperature, the charge of a small superconducting island is quantized in units of $2 e$ if the superconducting energy gap $\Delta$ is larger than the charging energy. ${ }^{10,16-18}$ In this paper, we restrict ourselves to the case where $\Delta>E_{c}$ so that quasiparticles can be neglected and the island charges can be expressed in terms of the number of excess Cooper pairs. A uniform gate voltage $V_{g}$ is applied to the islands by means of gate capacitors with capacitance $C_{g}$. Assuming $C_{g} \ll C$, the applied voltage induces a charge $C_{g} V_{g}$ on each island. In analogy with the magnetic frustration, we define a dimensionless charge frustration $n_{g}=C_{g} V_{g} / 2 e$ that describes the influence of the gate voltage. The two loops are subject to an externally applied flux $\Phi$ and 
the magnetic frustration is $f=\Phi / \Phi_{0}$, where $\Phi_{0}$ is the superconducting flux quantum. The sample is thus symmetric with respect to charge and magnetic frustration. The Josephson energy $E_{J}$ may be renormalized because of the electromagnetic environment and the finite charging energy of the islands. ${ }^{10,15}$

The Hamiltonian of the array $H=H_{\mathrm{el}}+H_{J}$ is the sum of the electrostatic Hamiltonian and the Josephson Hamiltonian. We consider here only the internal degrees of freedom of the array and do not take into account the bias circuit. The charge variables describing the state of the system are the numbers $n_{i}$ of excess Cooper pairs on the islands and the number $k$ of Cooper pairs having passed through the circuit. ${ }^{19}$ These numbers can themselves be expressed as a function of the set $\left\{k_{l}\right\}$ describing the number of Cooper pairs having tunneled through each junction $l$ of the circuit. Here $k=\left(k_{1}+k_{2}+k_{3}+k_{4}\right) / 2$ where the junctions are labeled according to the notations introduced in Fig. 1. The set of $\mathbf{n}=\left(n_{1}, n_{2}\right)$ and $k$ forms the so-called charge configuration of the array. The number of Cooper pairs, $n_{i}$, and the phase $\phi_{i}$ of the superconducting wave function of the islands are conjugate variables that satisfy $\left[\phi_{i}, n_{i}\right]=i$. Similarly, the difference $\delta$ between the superconducting phases of the leads and the charge flow index $k$ do not commute, $[\delta, k]=i$. Experimentally, arrays with $E_{J} \sim E_{c}$ are usually dc-current biased with a low-impedance electromagnetic environment. Therefore the phase difference $\delta$ is a classical variable ${ }^{20}$ while the phases $\phi_{i}$ and the numbers $n_{i}$ are quantum variables. Note that the role of the external phase difference and the flux in the two cells may be described in the same way by connecting the leads of the array and applying a magnetic flux to the loop formed in this way. We assume that the array will be probed by a current source, and following Ref. 2 we choose the basis of states $|\mathbf{n}, \delta\rangle$ to describe the array.

If the offset charges are compensated, the electrostatic energy of the array at zero bias voltage is given by

$$
E_{\mathrm{el}}(\mathbf{n})=\frac{1}{2}(2 e)^{2} \sum_{i, j}\left(n_{i}-n_{g}\right) C_{i j}^{-1}\left(n_{j}-n_{g}\right),
$$

where $C_{i j}^{-1}$ denotes the matrix element of the inverse capacitance matrix. On the other hand, the Josephson Hamiltonian couples states with different charge configurations but it is usually written in terms of the island phases as $H_{J}=-E_{J} \Sigma_{(p q)} \cos \left(\phi_{p}-\phi_{q}-A_{p q}\right)$, where the sum is over nearest-neighbor sites including the leads, and where $A_{p q}=2 \pi / \Phi_{0} \int_{p}^{q} \mathbf{A} \cdot d \mathbf{l}$, A being the vector potential. The Josephson Hamiltonian can be written in the charge representation in the following way. Each term of the sum in the Hamiltonian $H_{J}$ describes the tunneling of Cooper pairs across one junction. Tunneling of one Cooper pair across junction $l$ is described by $k_{l} \rightarrow k_{l}+\epsilon_{l}$, where $\epsilon_{l}= \pm 1$, and has two effects on the charge degrees of freedom $\mathbf{n}$ and $k$ of the array. It changes the charge configuration $\mathbf{n}\left(k_{l}\right)$ into $\mathbf{n}\left(k_{l}+\epsilon_{l}\right)$ but it also shifts the charge flow index $k$ (see above) by a quantity $\epsilon_{l}(\Delta k)_{l}$ given by $\epsilon_{l}(\Delta k)_{l}$ $=k\left(k_{l}+\epsilon_{l}\right)-k\left(k_{l}\right)$. We describe the first effect by a transition between two states while the change in $k$ is described by a phase factor $\exp \left[-i \epsilon_{l}(\Delta k)_{l} \delta\right]$. In the charge representation the Josephson Hamiltonian becomes

$$
\begin{aligned}
H_{J}= & -\sum_{l} E_{J} / 2\left\{\left|\mathbf{n}\left(k_{l}+\epsilon_{l}\right), \delta\right\rangle\left\langle\mathbf{n}\left(k_{l}\right), \delta\right| \exp \left[-i \epsilon_{l}(\Delta k)_{l} \delta\right]\right. \\
& \left.\times \exp \left(-i \epsilon_{l} A_{l}\right)+\text { H.c. }\right\},
\end{aligned}
$$

where the sum is over the junctions and where $\epsilon_{l} A_{l}$ is the line integral of the vector potential over junction $l$ associated with the tunnel event $k_{l} \rightarrow k_{l}+\epsilon_{l}$. Expressing the Josephson Hamiltonian $H_{J}$ in the basis of charge states $|\mathbf{n}, \delta\rangle$, the full Hamiltonian reads

$$
\begin{aligned}
H= & \sum_{\mathbf{n}} E_{\mathrm{el}}(\mathbf{n})|\mathbf{n}, \delta\rangle\langle\mathbf{n}, \delta| \\
& -\sum_{\langle\mathbf{n}, \mathbf{m}\rangle} E_{J} / 2\left[|\mathbf{m}, \delta\rangle\langle\mathbf{n}, \delta|\left(\sum_{\left\{l_{\mathbf{n}} \mathbf{m}\right.} \mathrm{e}^{-i \Phi_{l}}\right)+\text { H.c. }\right] .
\end{aligned}
$$

Here $\Sigma_{\langle\mathbf{n}, \mathbf{m}\rangle}$ denotes a sum over configurations which differ by the tunneling of one Cooper pair and $\{l\}_{\mathbf{n m}}$ is the set of all the single Cooper pair tunnel events which change the charge configuration from $\mathbf{n}$ to $\mathbf{m}$. The effect of the magnetic frustration is contained in the phase component $\Phi_{l}=\epsilon_{l}(\Delta k)_{l} \delta+\epsilon_{l} A_{l}$.

The eigenvectors $|\psi\rangle$ of the Hamiltonian $H$ are superpositions of charge states $|\psi\rangle=\Sigma_{\mathbf{n}} \alpha_{\mathbf{n}}\left(n_{g}, f, \delta\right)|\mathbf{n}, \delta\rangle$ and the eigenenergies form bands that are $2 \pi$ periodic in $\delta$. As a consequence of the charge quantization on the islands and the flux quantization in the loops, the ground state energy is periodic in both $n_{g}$ and $f$ with period 1 . We restrict ourselves to the intervals $0 \leqslant n_{g} \leqslant 1,0 \leqslant f \leqslant 1$ and we perform the calculation numerically using five possible numbers of Cooper pairs per island, i.e., $n_{i}=0, \pm 1, \pm 2$. With a typical value $E_{J} / E_{c}=0.2$, taking into account more charge configurations does not significantly change the results. The critical current $I_{c}$ of the array follows from the derivative of the ground state energy $E_{\text {gr }}$ with respect to the phase difference of the leads as $I_{c}=\left(2 \pi / \Phi_{0}\right) \max \left\{\partial E_{\mathrm{gr}} / \partial \delta\right\}$. The shape and the amplitude of the bands depend on the induced charge and the applied flux and so does the critical current.

As shown in Fig. 2, the two frustrations have a dual influence on the critical current, reflecting the duality between charge and flux in the array and the symmetry of the circuit. In fact, they act separately on the ground state of the system. The charge frustration $n_{g}$ affects the diagonal terms in the Hamiltonian $H$ by changing the electrostatic energy spectrum while the magnetic frustration $f$ affects the off-diagonal terms by modifying the coupling energy between the charge states. The lowest critical current as a function of $n_{g}$ is obtained at $n_{g}=0$ when the difference in electrostatic energy between the energetically most probable configuration and the other configurations is maximum. As a result the amplitude of the energy band associated with the ground state is minimized. At finite charge frustration, the energy difference between the charge states building the ground state of the system becomes smaller and the critical current is higher. At zero magnetic flux, the critical current as a function of $n_{g}$ displays two peaks due to the presence of two islands. This is reminiscent of the charge frustration dependence of the electrostatic energy which mainly defines the ground state energy when $E_{J} \ll E_{c}$. Here the position of the maxima of the critical current is not only set by the fundamental symmetries 

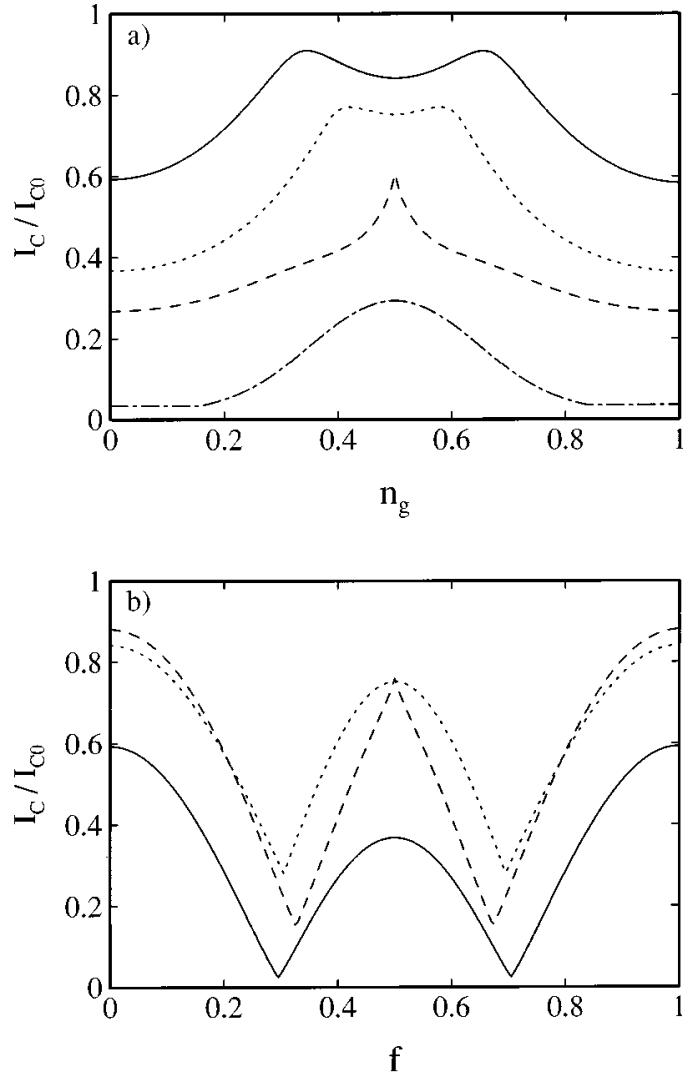

FIG. 2. (a) Critical current vs charge frustration for different values of the magnetic frustration, $f=0$ (solid line), $f=0.3$ (dashdotted), $f=0.4$ (long dashed line), and $f=0.5$ (short dashed line). (b) Critical current versus magnetic frustration for different values of the charge frustration, $n_{g}=0$ (solid line), $n_{g}=0.4$ (long dashed line), and $n_{g}=0.5$ (short dashed line). The ratio of the Josephson coupling energy $E_{J}$ and the charging energy $E_{c}$ is $E_{J} / E_{c}=0.2$. The current is normalized to $I_{C 0}=(2 e / \hbar) E_{J}$.

of the circuit like in a superconducting double junction but reflects the quantum nature of the ground state. If no charge frustration is applied [see Fig. 2(b)], the largest critical current is reached at $f=0$ when the coupling energy is maximized for all the transitions between charge states. In terms of Cooper pair tunneling, at small magnetic frustration the symmetry between the upper and lower island of the circuit is broken and the critical current is reduced.

Nevertheless, the critical current versus $f$ clearly resembles the magnetic diffraction pattern of a classical Josephson junction array. In an array with $E_{J} \gg E_{c}$, the dips in the diffraction pattern correspond to the entrance of a vortex. Classically, the definition of a vortex is that following a closed contour around the center of the vortex the sum of the phase differences should be $2 \pi$, when the phase differences are restricted to the interval $[-\pi, \pi]$. Due to the quantum fluctuations of the island phases, a vortex center can no longer be defined in a quantum array. In order to compare our circuit with its classical counterpart, we plot the ground state energy of the whole system as a function of the phase difference $\delta$ and the magnetic frustration $f$ as shown in Fig. 3 . In the $f, \delta$ plane the ground state energy displays a local minimum centered at $f=0.5$ and $\delta=\pi$. When sweeping $f$ from 0 to 0.5 the minimum of $E_{\mathrm{gr}}$ jumps once from $\delta=0$ to

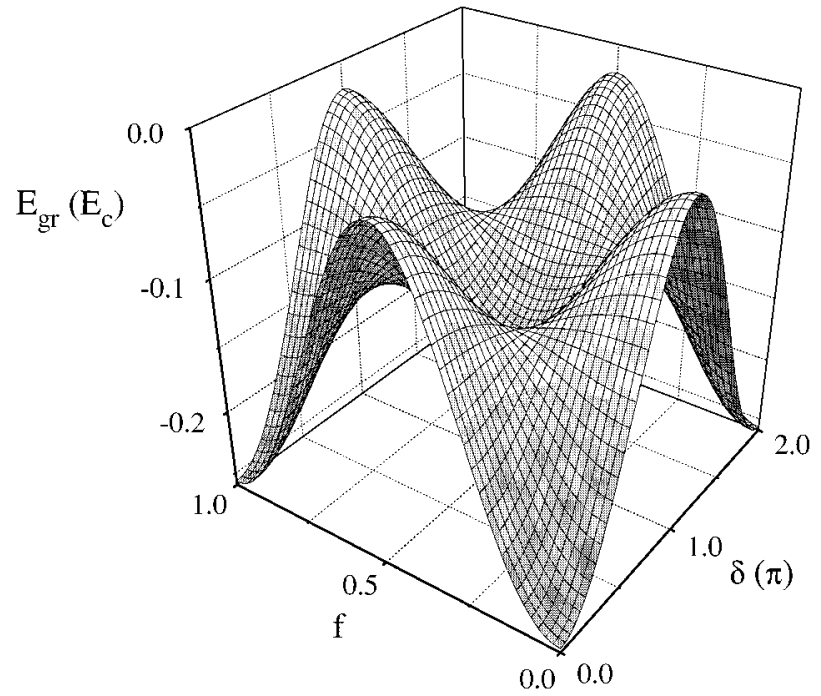

FIG. 3. Ground state energy vs frustration $f$ and phase difference of the leads $\delta$ at $n_{g}=0$ and $E_{J} / E_{c}=0.2$.

$\delta=\pi$ as it does when a vortex enters the array in the classical limit. More precisely, this discrete step occurs when the ground state energy $E_{\mathrm{gr}}$ crosses a saddle point in the $f, \delta$ plane. Similarly, the dips in the critical current [see Fig. 2(b)] correspond to a discontinuous change in the value of $\delta$ giving the maximum supercurrent. The ground state energy of the system exhibits the same structure as the one associated with the presence of a vortex in a classical array. The Josephson coupling energy that builds the supercurrent is also strong enough to build local minima in the ground state energy of the system despite the presence of charging effects. Further calculations, which we have performed on a quantum array consisting of four cells, confirm this result.

To address the relevance of a quantum vortex description of our circuit, we also calculate the expectation values of the current through the junctions. A vortex in a classical Josephson junction array is indeed characterized by a circulating current. To do so we set the net current to zero and compute $\left\langle I_{i}\right\rangle=-e\left\langle\partial k_{i} / \partial t\right\rangle$ for each junction of the circuit. In Fig. 4, we plot the current in one cell of the array as a function of

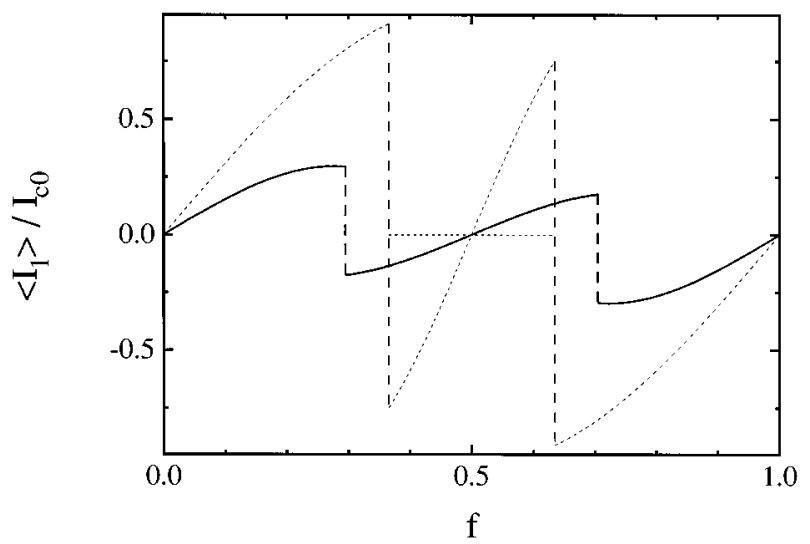

FIG. 4. Expectation value of the current through the upper left junction of the circuit as a function of the magnetic frustration $f$ for $E_{J} / E_{c}=0.2$ (solid line) and for $E_{J} \gg E_{c}$ (dotted line). The net current through the circuit is set to zero. 
the magnetic frustration $f$ in both the quantum and classical regimes. In the latter case, a low magnetic field induces the same current in the two loops. At $f=0.365$, a vortex enters the array and breaks the symmetry of the current distribution between the two cells. The ground state is then doubly degenerate for a vortex in the left-hand cell or in the right-hand cell. In the quantum regime, this degeneracy is lifted and the expectation value of the loop current is the same in both cells as shown in Fig. 4. Consequently, the expectation value of the current through the middle junction of the array is zero as we find numerically. The loop current $\left\langle I_{1}\right\rangle$, however, still exhibits two sudden changes in sign occurring at defined values of the frustration. One can interpret such a discrete event as the entrance of a quantum vortex in the array and the current distribution in the quantum regime as a superposition of the two current distributions associated with the two classical degenerate states. An important feature here is that the current steps are not rounded in the quantum regime. This is directly related to the presence of a local minimum in the ground state energy (see Fig. 3) and to the classical dynamics of the phase difference $\delta$. It implies that there is no quantum coherent coupling between the state without a vortex and the states with one vortex. In other words, in this model, a vacuum cannot play the role of a reservoir of vortices. Note that the case of charges is different since the superconducting leads play the role of reservoirs of Cooper pairs.

At $f=0$, we notice indeed that the charge frustration $n_{g}$ does not shift the value of $\delta$ at which the ground state energy is minimum. The peaks in the critical current as a function of $n_{g}$ are not associated with a discontinuity of $\delta$. In the presence of both charge and magnetic frustration the behavior of the critical current becomes more complex. The shape and depth of the well appearing in the $f, \delta$ plane depend on the charge frustration, yielding a gate voltage dependence of the magnetic diffraction pattern. When applying a magnetic frustration, the distance between the two peaks of the critical current as a function of $n_{g}$ becomes smaller as shown in Fig. 2(a). By breaking the symmetry between the upper and lower islands of the array, the magnetic frustration reduces the number of charge configurations involved in the ground state at low and high $n_{g}$ and shifts the peaks towards $n_{g}=0.5$.

We would like to point out that the critical current is a probe of the equilibrium properties of the array regardless of the bias circuitry. In practice, the $I-V$ characteristic and therefore the maximum supercurrent of a small Josephson junction array are determined by both the internal properties of the system and the electromagnetic environment. In the case of a double junction, the relationship between the maximum measured supercurrent and the critical current is complicated and strongly dependent on the impedance of the electromag- netic environment. Nevertheless, they both present qualitatively the same gate voltage dependence. ${ }^{2,4}$ Moreover, nonequilibrium measurements, i.e., at finite voltage, ${ }^{3,15}$ also reveal the quantum nature of the device. If the quantum array is current biased, the voltage is set by the dynamics of the phase difference of the leads that is influenced by the electromagnetic environment of the circuit. Since the static properties of the phase $\delta$ depend on the two frustrations $n_{g}$ and $f$, it is likely that its dynamics would exhibit the same features despite the effect of the environment. For small voltages, the $I-V$ characteristic of the quantum array would be $2 e$ periodic in the gate-induced charge and this modulation would be larger if the magnetic frustration $f$ is such that the system lies in a dip of the magnetic frustration pattern. In the phase description of a quantum array, the measured voltage across the circuit reflects the dynamics of quantum vortices in the array. The gate voltage dependence predicted by the charge description is therefore formally equivalent to the description in terms of vortex interference. In terms of Cooper pairs, the $I-V$ characteristic at low voltages observed in quantum arrays would be described as overlapping resonances occurring at finite voltages. The width of the resonances due to higher-order resonant Cooper pair tunneling depends on the Josephson coupling energy. The bias voltage at which these resonances occur depends on the gate voltage. Other resonances that can occur at finite voltage are the selfinduced Shapiro steps. The bias voltage at which these resonances occur does not depend on the gate voltage. The Shapiro steps appear when the Josephson frequency coincides with a resonant frequency of the impedance of the electromagnetic environment. ${ }^{22}$ For large arrays the number of resonances will also be large, closely spaced, and the individual structure of the resonances in the $I-V$ characteristic will be invisible. However, for smaller arrays in the quantum regime the individual resonances can be observed again. $3,15,21$

In conclusion, using the charge representation we have derived the equilibrium behavior of a small Josephson junction array as a function of induced charge and applied magnetic flux. Our model shows that the coherent superposition of states forming the ground state of the system and therefore the critical current can be tuned by both the charge and the magnetic frustration. We have shown that the notion of a vortex is compatible with a charge representation and appears naturally when introducing a finite magnetic frustration.

We would like to thank D. Esteve for helpful comments. We would also like to acknowledge the Dutch Foundation for Research on Matter (F.O.M.) for financial support. One of us (P.L.) acknowledges support of the European Economic Community under Contract No. ERBCHBICT941052.
${ }^{1}$ W. J. Elion, J. J. Wachters, L. L. Sohn, and J. E. Mooij, Phys. Rev. Lett. 71, 2311 (1993).

${ }^{2}$ P. Joyez, P. Lafarge, A. Filipe, D. Esteve, and M. H. Devoret, Phys. Rev. Lett. 72, 2458 (1994).

${ }^{3}$ D. B. Haviland, Y. Harada, P. Delsing, C. D. Chen, and T. Claeson, Phys. Rev. Lett. 73, 1541 (1994).
${ }^{4}$ M. Matters, W. J. Elion, and J. E. Mooij, Phys. Rev. Lett. 75, 721 (1995).

${ }^{5}$ R. Fazio and G. Schön, Phys. Rev. B 43, 5307 (1991).

${ }^{6}$ B. J. van Wees, Phys. Rev. B 44, 2264 (1991).

${ }^{7}$ U. Geigenmuller and G. Schön, Physica B 165 \& 166, 941 (1990). 
${ }^{8}$ D. V. Averin and K. K. Likharev, in Mesoscopic Phenomena in Solids, edited by B. L. Al'tschuler, P. Lee, and R. Webb (Elsevier, Amsterdam, 1991), Chap. 6.

${ }^{9}$ Alec Maassen van den Brink, Gerd Schön, and L. J. Geerligs, Phys. Rev. Lett. 67, 3030 (1991).

${ }^{10}$ K. A. Matveev, M. Gisselfält, L. I. Glazman, M. Jonson, and R. I. Shekter, Phys. Rev. Lett. 70, 994 (1993).

${ }^{11}$ T. A. Fulton, P. L. Gammel, D. J. Bishop, and L. N. Dunkleberger, Phys. Rev. Lett. 63, 1307 (1989).

${ }^{12}$ L. J. Geerligs, V. F. Anderegg, J. Romijn, and J. E. Mooij, Phys. Rev. Lett. 65, 377 (1990).

${ }^{13}$ M. T. Tuominen, J. M. Hergenrother, T. S. Tighe, and M. Tinkham, Phys. Rev. Lett. 69, 1997 (1992).

${ }^{14}$ T. M. Eiles and J. M. Martinis, Phys. Rev. B 50, 627 (1994).

${ }^{15}$ For a review see P. Joyez, Ph.D. thesis, Université Paris 6, 1995.
${ }^{16}$ D. V. Averin and Yu. V. Nazarov, Phys. Rev. Lett. 69, 1993 (1992).

${ }^{17}$ F. W. J. Hekking, L. I. Glazman, K. A. Matveev, and R. I. Shekter, Phys. Rev. Lett. 70, 4138 (1993).

${ }^{18}$ P. Lafarge, P. Joyez, D. Esteve, C. Urbina, and M. H. Devoret, Nature 365, 422 (1993).

${ }^{19}$ D. Esteve, in Single Charge Tunneling, edited by H. Grabert and M. H. Devoret (Plenum, New York, 1992), Chap. 3.

${ }^{20}$ G.-L. Ingold, H. Grabert, and U. Eberhardt, Phys. Rev. B 50, 395 (1994).

${ }^{21}$ W. J. Elion, Ph.D. thesis (Delft University Press, Delft, The Netherlands, 1995).

${ }^{22}$ T. Holst, D. Esteve, C. Urbina, and M. H. Devoret, Phys. Rev. Lett. 73, 3455 (1994). 